\title{
Deep Learning Approach for Enhanced Detection of Surface Plasmon
}

\author{
Scattering
}

Gwiyeong Moon*, Taehwang Son*, Hongki Lee, and Donghyun Kim ${ }^{\dagger}$

School of Electrical and Electronic Engineering

Yonsei University, Seoul, Korea, 120-749

\section{SUPPORTING INFORMATION}

- S1

- Table S1

- Table S2

- Figure S2

- Figure S3

- Table S3

- Figure S4

- Figure S5
Assessment of experimental signal-to-noise ratio $S_{N}$

Accuracy for monodisperse scatterers based on the conventional

reconstruction method

Accuracy for monodisperse scatterers based on the deep learning method SPM images that were incorrectly predicted by the deep learning method Enhancement factor $(E F)$ for each value of $S_{N}$

Accuracy for didisperse scatterers based on the deep learning method Repeated prediction of the number of scatterers after randomly removing scatterers and obstructors in the didisperse mixture

Effect of deep learning on the smallest distance of resolvable scatterers

\footnotetext{
* These authors contributed equally to this work.

† Corresponding author: kimd@yonsei.ac.kr
} 


\section{S1 Assessment of experimental signal-to-noise ratio $S_{N}$}

The following figure (Figure S1.1) presents SP scattering images that were experimentally acquired of a single scatterer in (a) and multiple scatterers in (b). X-shaped patterns near the high-contrast area are formed when a bead is slightly out of focus [Jiang, Y.; Wang, W. Point spread function of objective-based surface plasmon resonance microscopy. Anal. Chem. 2018, 90, 9650-9656].

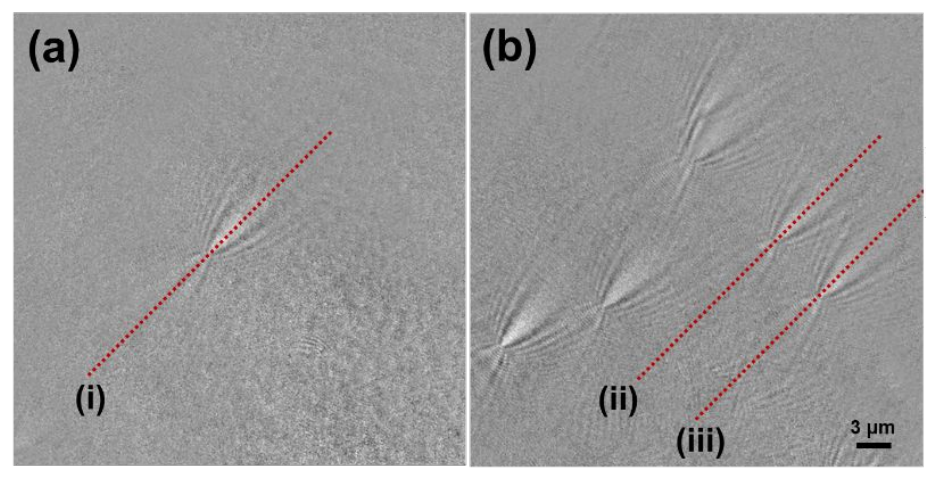

Figure S1.1 Experimental scattering images: (a) single scatterer and (b) multiple scatterers.

In order to find an SNR that corresponds to the experimental conditions, SP scattering images were obtained by simulation. Simulation results were then compared to match experimental values. Theoretical SP scattering images are as follows:

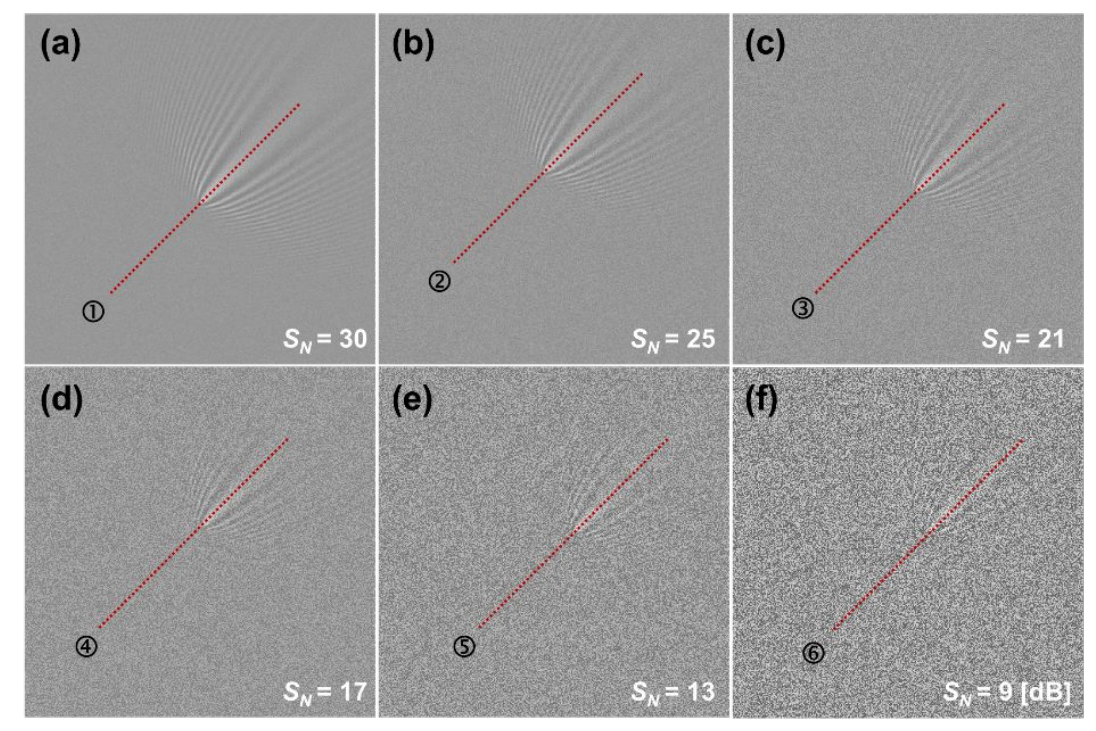

Figure S1.2 Theoretical SP scattering images: $S_{N}=$ (a) 30, (b) 25, (c) 21, (d) 17, (e) 13, and (f) $9 \mathrm{~dB}$.

For quantitative comparison of theoretical and experimental SNR in the surface plasmon scattering images, the intensity profile along the red line (dotted) in the images presented in Figures S1.1 and 2 was plotted in the following figure. 


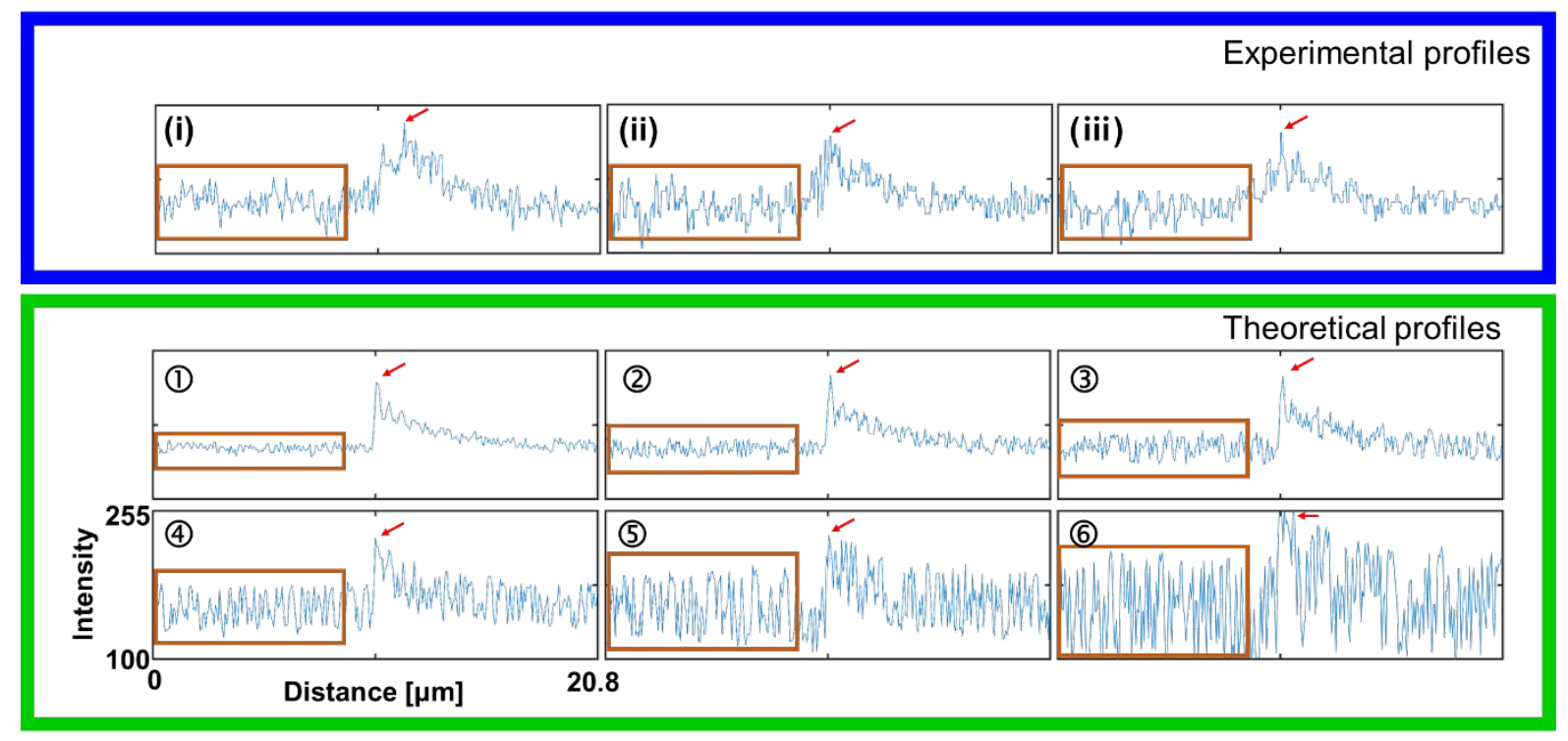

Figure S1.3 Intensity profiles along the red lines (dotted) of Figures S1.1 and 2: (i-iii) experimental plots vs. (1)(6) for theoretical profiles.

In the profiles presented in Figure S1.3, (i-iii) correspond to experimental images, while (1)-(6) were obtained from simulated ones with $S_{N}=30 \sim 9 \mathrm{~dB}$. In these profiles, maximum signal intensity obtained in the high contrast region of surface plasmon scattering patterns is marked by the red arrows). The profiles representing the background is shown in the orange box. Experimental SNR of surface plasmon scattering was found by comparing the noise power $\left(\sigma_{N}^{2}\right)$ in the background between experimental and theoretical results. Here, $\sigma_{N}^{2}=$ $\sum_{n=1}^{N}(f(n)-m)^{2} / N$ where $f, N$ and $m$ represent the signal in each orange box, signal length, and the average. The noise power is shown below in Figure S1.4:

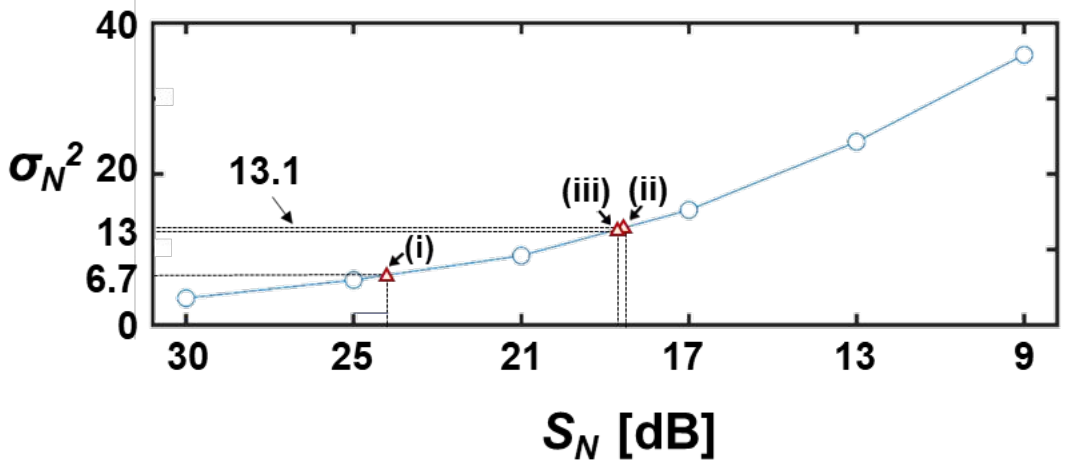

Figure S1.4 Noise power with respect to the image signal-to-noise ratio $S_{N}$. Experimental noise power is shown in triangles of (i-iii).

In the above figure, the blue line presents the noise power $\left(\sigma_{N}^{2}\right)$ from simulation results corresponding to $S_{N}=30$ $\sim 9 \mathrm{~dB}$. Experimental noise power is shown in triangles: $\sigma_{N}^{2}=6.7,13.1$, and 13 (unitless) for (i), (ii), and (iii). From the relationship, the noise level of the experimental images can be found to be between $S_{N}=18.5$ and 24.1 $\mathrm{dB}$. While this is an estimate using three sample images, we believe this to be a reasonable range to the first order. 
Table S1 Accuracy for monodisperse scatterers based on the conventional reconstruction method

\begin{tabular}{c|c|c|c|c|c|c|c|c}
\hline \multirow{2}{*}{$\boldsymbol{N}$} & \multicolumn{7}{c}{$\boldsymbol{\boldsymbol { S } _ { N } [ \mathbf { d B } ]}$} \\
\cline { 2 - 9 } & $\mathbf{3 0}$ & $\mathbf{2 5}$ & $\mathbf{2 1}$ & $\mathbf{1 7}$ & $\mathbf{1 3}$ & $\mathbf{9}$ & $\mathbf{5}$ & $\mathbf{1}$ \\
\hline $\mathbf{1}$ & 0.995 & 0.993 & 0.992 & 0.989 & 0.982 & 0.965 & 0.946 & 0.923 \\
$\mathbf{2}$ & 0.884 & 0.863 & 0.864 & 0.866 & 0.880 & 0.846 & 0.763 & 0.277 \\
$\mathbf{3}$ & 0.698 & 0.664 & 0.672 & 0.635 & 0.590 & 0.455 & 0.210 & 0.014 \\
$\mathbf{4}$ & 0.247 & 0.239 & 0.211 & 0.197 & 0.147 & 0.082 & 0.027 & 0.002 \\
$\mathbf{5}$ & 0.027 & 0.023 & 0.029 & 0.016 & 0.011 & 0.006 & 0.001 & 0.000 \\
$\mathbf{6}$ & 0.002 & 0.003 & 0.001 & 0.001 & 0.001 & 0.000 & 0.000 & 0.000 \\
$\mathbf{7}$ & 0.000 & 0.000 & 0.000 & 0.000 & 0.000 & 0.000 & 0.000 & 0.000 \\
$\mathbf{8}$ & 0.000 & 0.000 & 0.000 & 0.000 & 0.000 & 0.000 & 0.000 & 0.000 \\
$\mathbf{9}$ & 0.000 & 0.000 & 0.000 & 0.000 & 0.000 & 0.000 & 0.000 & 0.000 \\
\hline
\end{tabular}

Table S2 Accuracy for monodisperse scatterers based on the deep learning method

\begin{tabular}{c|c|c|c|c|c|c|c|c}
\hline \multirow{2}{*}{$\boldsymbol{N}$} & \multicolumn{7}{c}{$\boldsymbol{S}_{\boldsymbol{N}}[\mathbf{d B}]$} \\
\cline { 2 - 9 } & $\mathbf{3 0}$ & $\mathbf{2 5}$ & $\mathbf{2 1}$ & $\mathbf{1 7}$ & $\mathbf{1 3}$ & $\mathbf{9}$ & $\mathbf{5}$ & $\mathbf{1}$ \\
\hline $\mathbf{1}$ & 1.000 & 1.000 & 1.000 & 1.000 & 1.000 & 1.000 & 1.000 & 0.998 \\
$\mathbf{2}$ & 0.976 & 0.974 & 0.978 & 0.978 & 0.977 & 0.945 & 0.871 & 0.805 \\
$\mathbf{3}$ & 0.956 & 0.963 & 0.947 & 0.929 & 0.839 & 0.795 & 0.616 & 0.451 \\
$\mathbf{4}$ & 0.905 & 0.895 & 0.873 & 0.801 & 0.729 & 0.613 & 0.463 & 0.337 \\
$\mathbf{5}$ & 0.823 & 0.800 & 0.771 & 0.697 & 0.545 & 0.495 & 0.343 & 0.259 \\
$\mathbf{6}$ & 0.762 & 0.724 & 0.636 & 0.592 & 0.439 & 0.373 & 0.286 & 0.239 \\
$\mathbf{7}$ & 0.645 & 0.543 & 0.513 & 0.477 & 0.367 & 0.289 & 0.245 & 0.205 \\
$\mathbf{8}$ & 0.501 & 0.492 & 0.358 & 0.358 & 0.289 & 0.246 & 0.202 & 0.164 \\
$\mathbf{9}$ & 0.397 & 0.303 & 0.301 & 0.229 & 0.238 & 0.201 & 0.148 & 0.137 \\
\hline
\end{tabular}

Figure S2 SPM images that are incorrectly predicted by the deep learning method

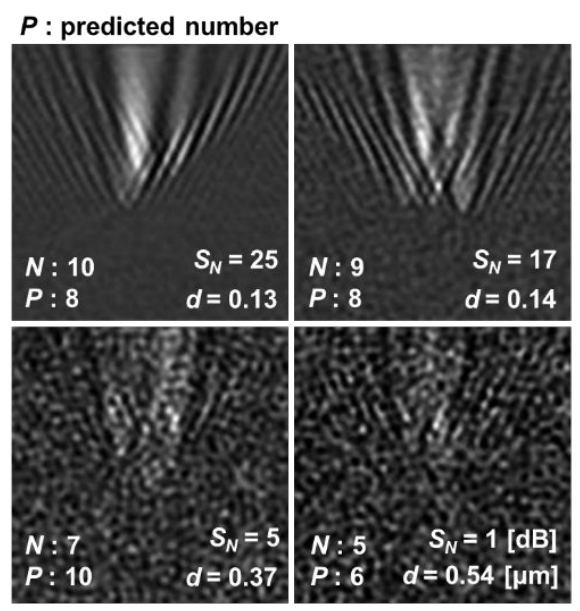

Figure S2. SPM images corresponding to the incorrect prediction by the deep learning approach. $P$ represents the predicted number of scatterers $(N)$. $S_{N}$ and $d$ are shown in $\mathrm{dB}$ and $\mu \mathrm{m}$. 
Figure S3 Enhancement factor $(E F)$ for each value of $S_{N}$

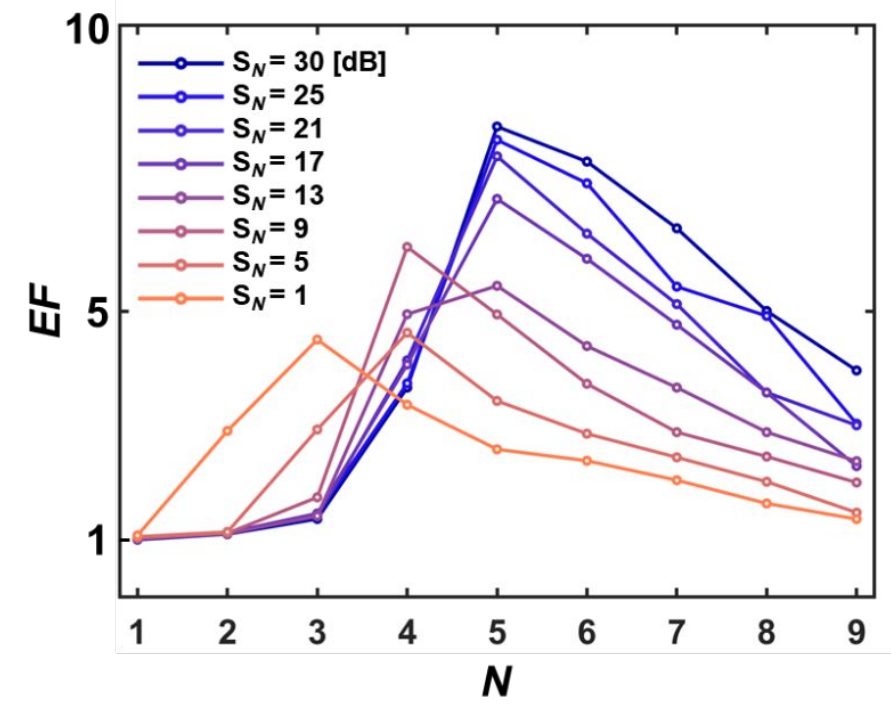

Figure S3. Enhancement factor $(E F)$ defines as $E F\left(S_{N}\right)=A_{D}\left(S_{N}\right) / \operatorname{Max}\left[A_{R}\left(S_{N}\right), 10 \%\right]$ is presented for each value of $S_{N} . N$ is the scatterer number. $E F$ is connected without interpolation. $E F$ is a function of $S_{N}$, while AEF is averaged over $S_{N}$.

Table S3 Accuracy for didisperse scatterers based on the deep learning method

\begin{tabular}{c|c|c|c|c|c|c|c|c}
\hline \multirow{2}{*}{$\boldsymbol{N}$} & \multicolumn{7}{c}{$\boldsymbol{S}_{\boldsymbol{N}}[\mathbf{d B}]$} \\
\cline { 2 - 8 } & $\mathbf{3 0}$ & $\mathbf{2 5}$ & $\mathbf{2 1}$ & $\mathbf{1 7}$ & $\mathbf{1 3}$ & $\mathbf{9}$ & $\mathbf{5}$ & $\mathbf{1}$ \\
\hline $\mathbf{1}$ & 0.991 & 0.989 & 0.987 & 0.985 & 0.977 & 0.982 & 0.931 & 0.927 \\
$\mathbf{2}$ & 0.913 & 0.905 & 0.905 & 0.883 & 0.866 & 0.795 & 0.667 & 0.475 \\
$\mathbf{3}$ & 0.755 & 0.722 & 0.669 & 0.641 & 0.561 & 0.479 & 0.445 & 0.359 \\
$\mathbf{4}$ & 0.563 & 0.548 & 0.515 & 0.490 & 0.473 & 0.385 & 0.393 & 0.273 \\
$\mathbf{5}$ & 0.551 & 0.479 & 0.435 & 0.435 & 0.319 & 0.302 & 0.255 & 0.187 \\
$\mathbf{6}$ & 0.499 & 0.408 & 0.396 & 0.349 & 0.274 & 0.240 & 0.229 & 0.196 \\
$\mathbf{7}$ & 0.443 & 0.377 & 0.337 & 0.289 & 0.269 & 0.234 & 0.205 & 0.172 \\
$\mathbf{8}$ & 0.409 & 0.331 & 0.283 & 0.263 & 0.218 & 0.206 & 0.172 & 0.131 \\
$\mathbf{9}$ & 0.263 & 0.241 & 0.203 & 0.187 & 0.167 & 0.159 & 0.117 & 0.116 \\
\hline
\end{tabular}




\section{Figure S4 Repeated prediction of the number of scatterers after randomly removing scatterers and obstructors in the didisperse mixture}

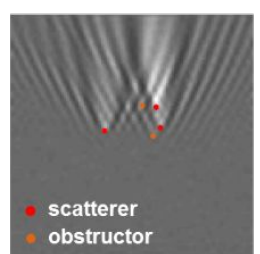

Remove scatterer or obstructor
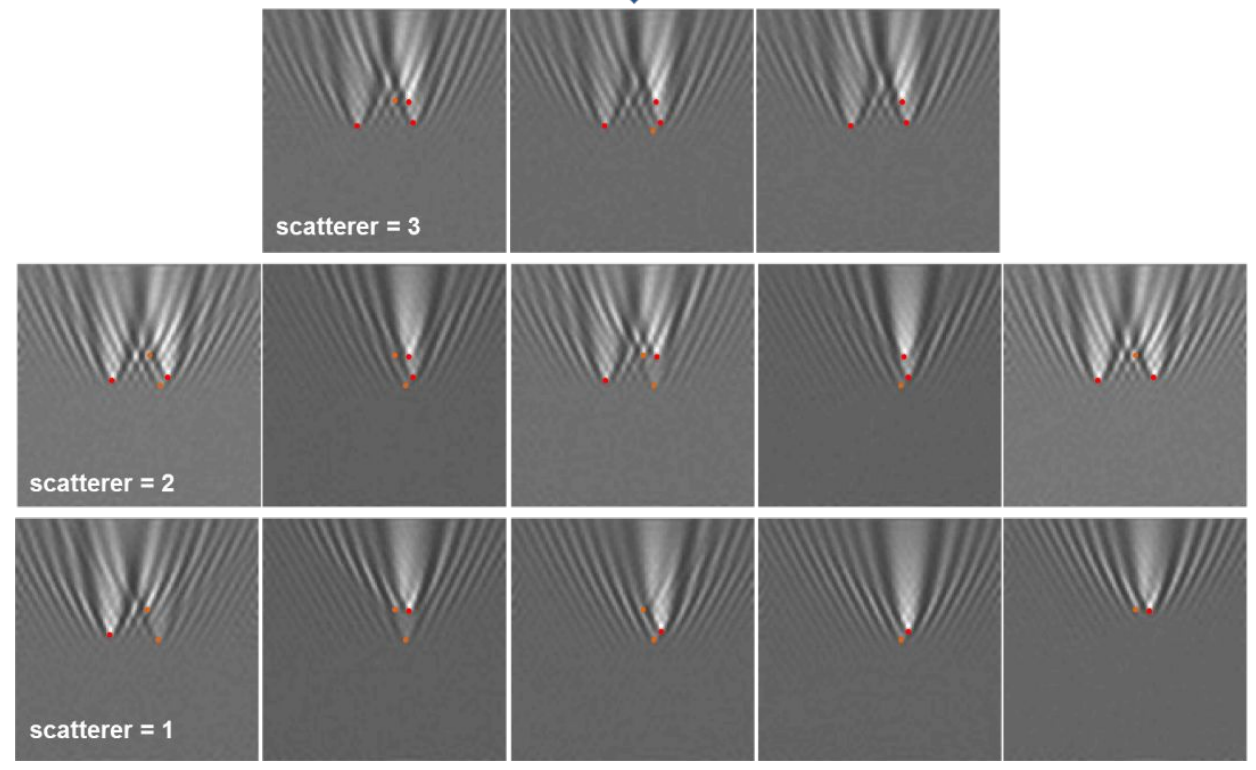

Figure S4. This presents a simple scenario to make sure that CNN would really count the number of scatterers, except for the obstructors. A SP scattering image was first generated with three scatterers and two obstructors (top row). The number of scatterers was predicted three. Next, three images were obtained by removing obstructors from the image of the top row (the second row), i.e., one obstructor was removed in the left, the other removed in the middle, and both removed in the right. The predicted number of scatterers turns out to be three. In a similar way, for each case of randomly removing one scatterer, there are four possibilities of obstructors (keeping all obstructors, removing one of the two, and removing both), therefore, 12 cases of having one scatterer removed in total. Out of these 12, five cases are presented in the third row. Likewise, there are 12 cases of randomly removing two scatterers, out of which we have presented five in the fourth row. For the cases of removing one and two scatterers randomly removed, the number of scatterers was predicted to be one and two, respectively. In other words, the results confirm that the number of obstructors does not affect the performance of CNN, while the number scatterers does. Obviously, in the presence of stronger noise, the performance of CNN predicting the number characteristics deteriorates. This, however, is not due directly to the $\mathrm{CNN}$, but to the noise. In this sense, we conclude that $\mathrm{CNN}$ extracts useful information such as the number of scatterers in an image in the presence of polydisperse obstructors. 
Figure S5 Effect of deep learning on the smallest distance of resolvable scatterers
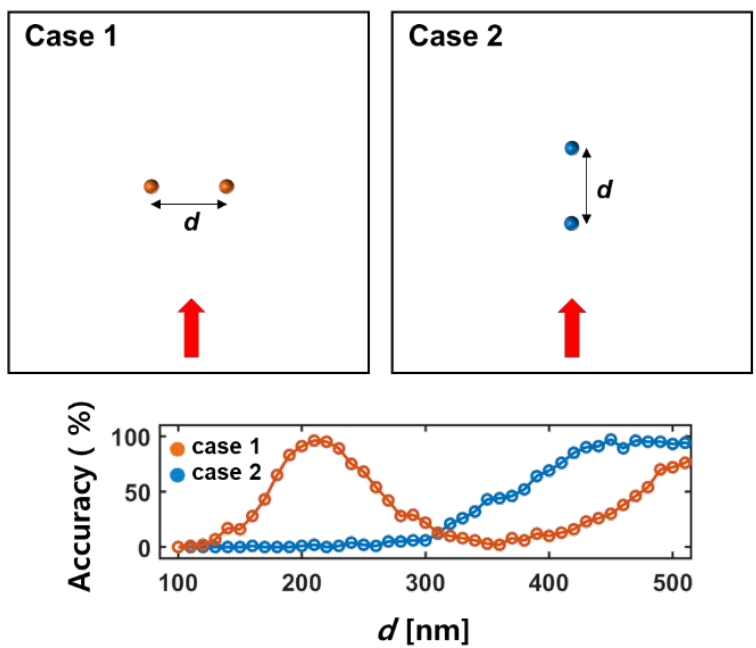
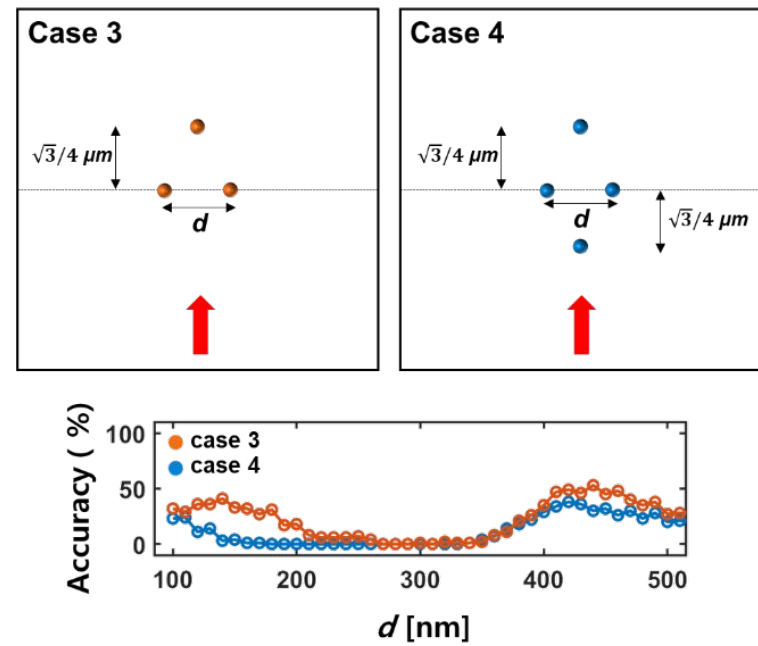

Figure S5. In order to address the effect of deep learning on the smallest distance of resolvable scatterers, we first define "being resolved" as two scatterers estimated to be separated with an accuracy higher than $50 \%$. Consider two scenarios. In one scenario, two scatterers are positioned such that the line between the two scatterers is perpendicular to the direction of propagation of plasmonic fields (Case 1). The direction of surface plasmon propagation is represented by a red arrow. In the other scenarios, two scatterers are positioned so that the line is parallel to the direction of SP propagation (Case 2). Inter-scatterer distance $(d)$ was varied from $d=100$ to 500 $\mathrm{nm}$ with a 10-nm interval. 100 images were generated for each $d$ where noise was added at $\mathrm{SN}=1 \mathrm{~dB}$. The results indicate that one may use $\mathrm{CNN}$ to resolve two scatterers separated by a shorter $d$ in Case 1 than in Case 2, i.e., the shortest $d$ corresponding to the $50 \%$ accuracy is $d_{\min } \approx 150 \mathrm{~nm}$ for Case 1 vs. $d_{\min } \approx 350 \mathrm{~nm}$ for Case 2 . This is attributed to the difference in the image contrast that depends on the distribution of scatterers with respect to the direction of SP waves. In other words, in Case 2, scattering field of a scatterer is fully enclosed by that of the other scatterer. In contrast, scattering patterns are somewhat independent in Case 1, making the process of resolving the two scatterers easier. However, the whole process is much more complicated due to the presence of near-field interference, which we believe is responsible for the decrease of accuracy in Case 1 between $d=220$ and $500 \mathrm{~nm}$. To show the complex nature of the estimation, more scenarios are considered. In Case 3: three scatterers are positioned, while Case 4 positions four scatterers. Like in the previous cases, inter-scatterer distance $(d)$ was varied from $d=100$ to $500 \mathrm{~nm}$. In Case 3 and 4, the accuracy in general decreased significantly compared to Case 1 and 2 , although the accuracy tends to be high near $d=100 \sim 200 \mathrm{~nm}$. Again, this may be an artifact arising from SP interference among scatterers. 